\title{
Relations Among Linear and Circular Polarization Parameters Measured in Canted Hydrometeors
}

\author{
A. R. JAMESON \\ Applied Research Corporation, Landover, MD 20785 \\ (Manuscript received 9 July 1986, in final form 10 April 1987)
}

\begin{abstract}
Parameters derived from measurements using circular and linear polarizations are mathematically interrelated. In this work this interdependence is investigated. It is shown that circular and linear polarization parameters share a common meteorological interpretation which is sensitive to both particle canting and the radar elevation angle.
\end{abstract}

\section{Introduction}

The two most frequently used polarizations in meteorological research are horizontal and vertical linear and left- and right-handed circular. The radiation backscattered by precipitation is related to the incident wave by a backscatter matrix S (Sinclair, 1948). In the absence of nonlinear propagation effects the backscatter matrices corresponding to different elliptical polarizations are linear transformations of one another (e.g., Maffett, 1968). The parameters associated with a particular polarization, however, are usually derived from the autocorrelations and cross-correlations between backscattered orthogonally polarized waves. Since these quantities can be written as products of elements of $\mathbf{S}$, parameters characteristic of one polarization are not simple linear transformations of those at another polarization. Nevertheless, regardless of the kind of polarization, the physics is the same. Therefore, a common framework should exist for interpreting parameters using any elliptical polarization. The challenge is to find a general interpretation that can still provide useful meteorological information.

For symmetric scatterers which are small with respect to the radar wavelength (Rayleigh-Gans scatterers), the polarization parameters depend in part upon the shapes of the particles. Since several scatterers usually occupy a radar sampling volume, the polarization measurements reflect the properties of the distributions of particle shapes. In particular, for spheroidal scatterers the polarization parameters should be related to the reflectivity-weighted mean axis ratio $R$ and the variance of the reflectivity weighted axis ratio distribution $\sigma_{r}{ }^{2}$. In addition the parameters are sensitive to the distribution of canting angles (the angle between local vertical and the direction of the axis of symmetry of the particle). Polarization parameters, therefore, are related. to the variance of the distribution of angles $\sigma_{\alpha}{ }^{2}$ and, in principle, the mean canting angle. This latter quantity, however, is usually thought to be very close to zero (Jameson, 1985).

Using circular polarization parameters, methods for estimating $R$ (McCormick, 1979; Jameson, 1983), $\sigma_{r}^{2}$ (Jameson, 1983), and $\sigma_{\alpha}^{2}$ (Hendry et al., 1987) have been suggested. Similarly $R$ (Jameson, 1983) and $\sigma_{\alpha}{ }^{2}$ (Jameson, 1985) can be estimated from linear polarization measurements.

The magnitude $\left(\rho_{L}\right)$ of the cross correlation between horizontal and vertical copolarized signals (that is, the polarization of the received and transmitted waves are identical) can be obtained by rapidly switching between these two orthogonal polarizations (Jameson and Mueller, 1985; Sachidananda and Zrnić, 1986). In a manner quite analogous to that for circular polarization, it is shown in this work that $\rho_{L}$ combined with differential reflectivity yields an estimate of $\sigma_{r}^{2}$. For Rayleigh-Gans spheroidal scatterers, therefore, the identical meteorological information can be extracted from either circular or linear polarization parameters.

\section{Relationships between linear and circular polariza- tion parameters \\ a. Autocorrelations and cross-correlations for linear and circular polarizations}

If $\mathbf{S}_{L}$ and $\mathbf{S}_{C}$ denote the backscatter matrices corresponding to linear and circular polarizations then

$$
\begin{aligned}
& \mathbf{s}_{L}=\left[\begin{array}{ll}
S_{H H} & S_{H V} \\
S_{V H} & S_{V V}
\end{array}\right] \\
& \mathbf{s}_{C}=\left[\begin{array}{ll}
S_{R R} & S_{R L} \\
S_{L R} & S_{L L}
\end{array}\right]
\end{aligned}
$$


where

$$
\begin{aligned}
& S_{R R}=\left(S_{V V}-S_{H H}-2 j S_{H V}\right) / 2 \\
& S_{L L}=\left(S_{V V}-S_{H H}+2 j S_{H V}\right) / 2 \\
& S_{R L}=S_{L R}=\left(S_{H H}+S_{V V}\right) / 2,
\end{aligned}
$$

and $j$ denotes the imaginary number $\sqrt{-1}$. The first and second subscripts identify the polarization state of the transmitted and received waves, respectively; $V, H$, $R$ and $L$ denote vertical linear, horizontal linear, righthanded circular and left-handed circular polarizations, respectively.

In general, the signals measured by radars result from the superposition of the waves scattered independently by all the hydrometeors in the illuminated volume. At each instant it follows for linear polarizations that

$$
\left.\begin{array}{l}
E_{H H}=C \sum_{i} S_{H H}^{i} \exp \left\{j\left(\psi_{i}+2 \phi_{H}^{i}\right)\right\} \\
E_{V V}=C \sum_{i} S_{V V}^{i} \exp \left\{j\left(\psi_{i}+2 \phi_{V}^{i}\right)\right\} \\
E_{H V}=C \sum_{i} S_{H V}^{i} \exp \left\{j\left(\psi_{i}+\phi_{H}^{i}+\phi_{V}^{i}\right)\right\} \\
E_{V H}=C \sum_{i} S_{V H}^{i} \exp \left\{j\left(\psi_{i}+\phi_{H}^{i}+\phi_{V}^{i}\right)\right\}
\end{array}\right\},
$$

while for circularly polarized waves

$$
\left.\begin{array}{l}
E_{R L}=\frac{C}{2} \sum_{i}\left[S_{H H}^{i} \exp \left\{j \phi_{H}{ }^{i}\right\}+S_{V V}^{i} \exp \left\{j \phi_{V}{ }^{i}\right\}\right] \exp \left\{j \psi_{i}\right\} \\
E_{R R}=\frac{C}{2} \sum_{i}\left[S_{V V}^{i} \exp \left\{j \phi_{V}{ }^{i}\right\}-S_{H H}^{i} \exp \left\{j \phi_{H}{ }^{i}\right\}-2 j S_{H V}^{i} \exp \left\{j\left(\phi_{H}{ }^{i}+\phi_{V}{ }^{i}\right)\right\}\right] \exp \left\{\psi_{i}\right\} \\
E_{L R}=\frac{C}{2} \sum_{i}\left[S_{H H}^{i} \exp \left\{j \phi_{H}{ }^{i}\right\}+S_{V V}^{i} \exp \left\{j \phi_{V}{ }^{i}\right\}\right] \exp \left\{j \psi_{i}\right\} \\
E_{L L}=\frac{C}{2} \sum_{i}\left[S_{V V}^{i} \exp \left\{j \phi_{V}{ }^{i}\right\}-S_{H H}^{i} \exp \left\{j \phi_{H}{ }^{i}\right\}+2 j S_{H V}^{i} \exp \left\{j\left(\phi_{H}{ }^{i}+\phi_{V}{ }^{i}\right)\right\}\right] \exp \left\{\psi_{i}\right\}
\end{array}\right\},
$$

where the summation is over all the particles in the sampling volume. $\psi_{i}=4 \pi \lambda / r_{i}$ is the phase position of the $i$ th scatterer at range $r_{i}$ from the radar operating at a wavelength $\lambda$, while $\phi_{i}$ denotes the propagation phase shift at $r_{i}$. For a short radar pulse length, the dependence of intensity on the distance to the center of the sampling volume is essentially constant and is included in the coefficient $C$. The illumination is assumed to be uniform for all polarizations.

With time radar signals fluctuate largely because of motion of the particles with respect to one another which causes random constructive and destructive interference among the waves backscattered from each particle. Polarization parameters, therefore, must be estimated from time averages of elements of autocorrelations and cross correlations of signals. After averaging, the random phases tend to cancel leaving only the mean values. If the averaging is long, it is often assumed that essentially all realizations of the ensemble of particles have been sampled so that the time average and ensemble average may be equated. From (3) and (4) the essential quantities can be written as

$$
\begin{aligned}
& \left|E_{R R}\right|^{2}=\frac{1}{4}\left\{\left|E_{H H}\right|^{2}+\left|E_{V V}\right|^{2}+4\left|E_{H V}\right|^{2}-2 \operatorname{Re}\left\langle E_{H H} E_{V V}^{*}\right\rangle+4 \operatorname{Im}\left\langle\left[\left(E_{H H}-E_{V V}\right) E_{H V}^{*}\right]\right\rangle\right\} \\
& \left|E_{L L}\right|^{2}=\frac{1}{4}\left\{\left|E_{H H}\right|^{2}+\left|E_{V V}\right|^{2}+4\left|E_{H V}\right|^{2}-2 \operatorname{Re}\left\langle E_{H H} E_{V V}^{*}\right\rangle-4 \operatorname{Im}\left\langle\left[\left(E_{H H}-E_{V V}\right) E_{H V}^{*}\right]\right\rangle\right\} \\
& \left|E_{R L}\right|^{2}=\left|E_{L R}\right|^{2} \\
= & \frac{1}{4}\left\{\left|E_{H H}\right|^{2}+\left|E_{V V}\right|^{2}+2 \operatorname{Re}\left\langle E_{H H} E_{V V}^{*}\right\rangle\right\} \\
\left\langle E_{R R} E_{R L}^{*}\right\rangle= & \left(\left|E_{R R}\right|^{2}\left|E_{R L}\right|^{2}\right)^{0.5} \nu_{R C} \\
= & \left(\left|E_{R R}\right|^{2}\left|E_{R L}\right|^{2}\right)^{0.5} \rho_{c} \exp \left(j \Phi_{c}\right) \\
= & \frac{1}{4}\left\{\left|E_{V V}\right|^{2}-\left|E_{H H}\right|^{2}-2 \operatorname{Im}\left\langle\left[\left(E_{H H}+E_{V V}\right) E_{H V}^{*}\right]\right\rangle+2 j\left(\operatorname{Im}\left\langle E_{H H} E_{V V}^{*}\right\rangle+\operatorname{Re}\left\langle\left[\left(E_{H H}+E_{V V}\right) E_{H V}^{*}\right]\right\rangle\right)\right\}
\end{aligned}
$$




$$
\begin{aligned}
\left\langle E_{L L} E_{L R}^{*}\right\rangle= & \left(\left|E_{L L}\right|^{2}\left|E_{L R}\right|^{2}\right)^{0.5} \nu_{L C} \\
= & \frac{1}{4}\left\{\left|E_{V V}\right|^{2}-\left|E_{H H}\right|^{2}+2 \operatorname{Im}\left\langle\left[\left(E_{H H}+E_{V V}\right) E_{H V}^{*}\right]\right\rangle+2 j\left(\operatorname{Im}\left\langle E_{H H} E_{V V}^{*}\right\rangle-\operatorname{Re}\left\langle\left[\left(E_{H H}+E_{V V}\right) E_{H V}^{*}\right]\right\rangle\right)\right\} \\
\left\langle E_{R R} E_{L L}^{*}\right\rangle & =\left(\left|E_{R R}\right|^{2}\left|E_{L L}\right|^{2}\right)^{0.5} \nu_{R L} \\
& =\frac{1}{4}\left(\left|E_{H H}\right|^{2}+\left|E_{V V}\right|^{2}-4\left|E_{H V}\right|^{2}-2 \operatorname{Re}\left\langle E_{H H} E_{V V}^{*}\right\rangle+4 j \operatorname{Re}\left\langle\left[\left(E_{H H}-E_{V V}\right) E_{H V}^{*}\right]\right\rangle\right\} \\
\left|E_{H H}\right|^{2} & =\frac{1}{4}\left\{\left|E_{R R}\right|^{2}+\left|E_{L L}\right|^{2}+4\left|E_{R L}\right|^{2}-4 \operatorname{Re}\left\langle E_{R R} E_{R L}^{*}\right\rangle-4 \operatorname{Re}\left\langle E_{L L} E_{L R}^{*}\right\rangle+2 \operatorname{Re}\left\langle E_{R R} E_{L L}^{*}\right\rangle\right\} \\
\left|E_{V V}\right|^{2} & =\frac{1}{4}\left\{\left|E_{R R}\right|^{2}+\left|E_{L L}\right|^{2}+4\left|E_{R L}\right|^{2}+4 \operatorname{Re}\left\langle E_{R R} E_{R L}^{*}\right\rangle+4 \operatorname{Re}\left\langle E_{L L} E_{L R}^{*}\right\rangle+2 \operatorname{Re}\left\langle E_{R R} E_{L L}^{*}\right\rangle\right\} \\
\left|E_{H V}\right|^{2} & =\frac{1}{4}\left\{\left|E_{R R}\right|^{2}+\left|E_{L L}\right|^{2}-2 \operatorname{Re}\left\langle E_{R R} E_{L L}^{*}\right\rangle\right\} \\
\left\langle E_{H H} E_{V V}^{*}\right\rangle & =\left(\left|E_{H H}\right|^{2}\left|E_{V V}\right|^{2}\right)^{0.5} \nu_{L} \\
& =\left(\left|E_{H H}\right|^{2}\left|E_{V V}\right|^{2}\right)^{0.5} \rho_{L} \exp \left(j \Phi_{L}\right) \\
& =\frac{1}{4}\left\{4\left|E_{R L}\right|^{2}-\left|E_{R R}\right|^{2}-\left|E_{L L}\right|^{2}-2 \operatorname{Re}\left\langle E_{R R} E_{L L}^{*}\right\rangle+4 j\left(\operatorname{Im}\left\langle E_{R L} E_{R R}^{*}\right\rangle+\operatorname{Im}\left\langle E_{L R} E_{L L}^{*}\right\rangle\right)\right\},
\end{aligned}
$$

where \langle\rangle denotes time averaging; $|E|^{2}=\left\langle E E^{*}\right\rangle$, while the complex number $\nu$ denotes various crosscorrelation functions; $\left\langle E_{H H} E_{V V}^{*}\right\rangle$ and $\left\langle E_{R R} E_{L L}^{*}\right\rangle$ must be formed from proper sequencing of alternating copolarizations (Jameson and Mueller, 1985; Sachidananda and Zrnić, 1986). For simplicity $E_{V H}$ is replaced by $E_{H V}$ as permitted by the reciprocity theorem (Saxon, 1955).

Some simplification of (5) is possible. First, $\left|E_{R R}\right|^{2}$ must equal $\left|E_{L L}\right|^{2}$ since $\left|S_{R R}\right|^{2}=\left|S_{L L}\right|^{2}$ so that $\operatorname{Im}\left\langle\left(E_{H H}\right.\right.$ $\left.\left.-E_{V V}\right) E_{H V}^{*}\right\rangle$ must be zero. Second, for RayleighGans scatterers the cross-correlations $\left\langle E_{H H} E_{H V}^{*}\right\rangle$ and $\left\langle E_{V V} E_{H V}^{*}\right\rangle$ will probably be very small since the large number of scatterers in a typical radar sampling volume will tend to guarantee that the contributions to the cross-polarization will be nearly symmetrically distributed with respect to the local true vertical. It follows then that

$$
\begin{aligned}
&\left|E_{R R}\right|^{2}=\left|E_{L L}\right|^{2} \\
&=\frac{1}{4}\left\{\left|E_{H H}\right|^{2}+\left|E_{V V}\right|^{2}+4\left|E_{H V}\right|^{2}-2 \operatorname{Re}\left\langle E_{H H} E_{V V}^{*}\right\rangle\right\} \\
& \nu_{R C}=\nu_{L C} \\
&=\frac{\left|E_{V V}\right|^{2}-\left|E_{H H}\right|^{2}+2 j \operatorname{Im}\left\langle E_{H H} E_{V V}^{*}\right\rangle}{\left(\left\{\left|E_{H H}\right|^{2}+\left|E_{V V}\right|^{2}+4\left|E_{H V}\right|-2 \operatorname{Re}\left\langle E_{H H} E_{V V}^{*}\right\rangle\right\} \times\left\{\left|E_{H H}\right|^{2}+\left|E_{V V}\right|^{2}+2 \operatorname{Re}\left\langle E_{H H} E_{V V}^{*}\right\rangle\right\}\right)^{0.5}} \\
&\left|E_{H H}\right|^{2}=\frac{1}{4}\left\{2\left|E_{R R}\right|^{2}+4\left|E_{R L}\right|^{2}-8\left(\left|E_{R R}\right|^{2}\left|E_{R L}\right|^{2}\right)^{0.5} \operatorname{Re}\left(\nu_{R C}\right)+2 \operatorname{Re}\left\langle E_{R R} E_{L L}^{*}\right\rangle\right\} \\
&\left|E_{V V}\right|^{2}=\frac{1}{4}\left\{2\left|E_{R R}\right|^{2}+4\left|E_{R L}\right|^{2}+8\left(\left|E_{R R}\right|^{2}\left|E_{L L}\right|^{2}\right)^{0.5} \operatorname{Re}\left(\nu_{R C}\right)+2 \operatorname{Re}\left\langle E_{R R} E_{L L}^{*}\right\rangle\right\} \\
&\left|E_{H V}\right|^{2}=\frac{1}{4}\left\{2\left|E_{R R}\right|^{2}-2 \operatorname{Re}\left\langle E_{R R} E_{L L}^{*}\right\rangle\right\} \\
&\left(\left|E_{H H}\right|^{2}\left|E_{V V}\right|^{2}\right)^{0.5} \nu_{L}=\frac{1}{4}\left\{4\left|E_{R L}\right|^{2}-2\left|E_{R R}\right|^{2}-2 \operatorname{Re}\left\langle E_{R R} E_{L L}^{*}\right\rangle+4 j\left(\operatorname{Im}\left\langle E_{R L} E_{R R}^{*}\right\rangle+\operatorname{Im}\left\langle E_{R R} E_{L L}^{*}\right\rangle\right)\right\}
\end{aligned}
$$


From (5) and (6) it is possible to formulate both circular and linear polarization parameters in terms of each other.

\section{b. The polarization parameters}

1) LINEAR POLARIZATION PARAMETERS FROM CIRCULAR POLARIZATION MEASUREMENTS

The parameters of interest are the differential reflectivity $\zeta$, the linear depolarization ratio $L$, and the linear copolarization cross-correlation $\nu_{L}$. Specifically,

$$
\begin{aligned}
\zeta & =\frac{\left|E_{H H}\right|^{2}}{\left|E_{V V}\right|^{2}} \\
L & =10^{(L D R / 10)} \\
& =\frac{\left|E_{H V}\right|^{2}}{\left|E_{H H}\right|^{2}} \\
\nu_{L} & =\rho_{L} \exp \left(j \Phi_{L}\right) \\
& =\frac{\left\langle E_{H H} E_{V V}^{*}\right\rangle}{\left(\left|E_{H H}\right|^{2}\left|E_{V V}\right|^{2}\right)^{0.5}} .
\end{aligned}
$$

Expressed in terms of circular polarization (7) becomes

$$
\begin{gathered}
\zeta=\frac{2+\Gamma\left\{1+\operatorname{Re}\left(\nu_{R L}\right)\right\}-4 \Gamma^{0.5} \operatorname{Re}\left(\nu_{R C}\right)}{2+\Gamma\left\{1+\operatorname{Re}\left(\nu_{R L}\right)\right\}+4 \Gamma^{0.5} \operatorname{Re}\left(\nu_{R C}\right)} \\
L=\frac{\Gamma\left\{1-\operatorname{Re}\left(\nu_{R L}\right)\right\}}{2+\Gamma\left\{1+\operatorname{Re}\left(\nu_{R L}\right)\right\}-4 \Gamma^{0.5} \operatorname{Re}\left(\nu_{R C}\right)} \\
\nu_{L}=\rho_{L} \exp \left(j \phi_{L}\right) \\
=\frac{2-\Gamma\left\{1+\operatorname{Re}\left(\nu_{R L}\right)\right\}+4 j \Gamma^{0.5} \operatorname{Im}\left(\nu_{R C}\right)}{\left(\left\{2+\Gamma\left[1+\operatorname{Re}\left(\nu_{R L}\right)\right]+4 \Gamma^{0.5} \operatorname{Re}\left(\nu_{R C}\right)\right\}\right.} \\
\left.\quad \times\left\{2+\Gamma\left[1+\operatorname{Re}\left(\nu_{R L}\right)\right]-4 \Gamma^{0.5} \operatorname{Re}\left(\nu_{R C}\right)\right\}\right)^{0.5}
\end{gathered}
$$

where the circular depolarization ratio is given by

$$
\begin{aligned}
\Gamma & =\frac{\left|E_{R R}\right|^{2}}{\left|E_{R L}\right|^{2}} \\
& =\frac{\left|E_{L L}\right|^{2}}{\left|E_{L R}\right|^{2}} \\
& =10^{(C D R / 10)}
\end{aligned}
$$

and $\nu_{R C}$ and $\nu_{R L}$ are defined by (5d) and (5f), respectively.

In contrast to a direct measurement of $\zeta$ using linear polarization, (8a) requires four quantities, namely powers in the co- and cross-polarized channels as well as two cross-correlations, $\nu_{R C}$ and $\nu_{R L}$. To compute $\nu_{R L}$ it is necessary to switch rapidly between right- and lefthanded circular polarizations. Alternatively, this quantity can probably be ignored since the estimate of $\zeta$ is only slightly affected as $\operatorname{Re}\left(\nu_{R L}\right)$ varies from 0 to 1. By setting $\operatorname{Re}\left(\nu_{R L}\right)$ to unity, Eq. (8a) becomes equivalent to the estimate of $\zeta$ derived by McCormick (1979).
The most serious limitation to estimating $\zeta$ from (8a) will be the bias introduced by propagation differential phase shift.

The other linear polarization quantity of particular interest is the canting parameter $\gamma$ (Jameson, 1985) which can be written as

$$
\begin{aligned}
\gamma & =\frac{\left|E_{H V}\right|^{2}}{\left|E_{H H}\right|^{2}+\left|E_{V V}\right|^{2}-2 \operatorname{Re}\left\langle E_{H H} E_{V V}^{*}\right\rangle} \\
& =\frac{L \zeta}{1+\zeta-2 \zeta^{0.5} \operatorname{Re}\left(\nu_{L}\right)} .
\end{aligned}
$$

For Rayleigh-Gans scatterers, $\operatorname{Re}\left(\nu_{L}\right) \approx \rho_{L}$ (Jameson and Mueller, 1985) so that a good estimate of $\gamma$ will be given by

$$
\xi(\gamma)=\frac{L \zeta}{1+\zeta-2 \zeta^{0.5} \rho_{L}} .
$$

Since $\rho_{L}$ is not affected by propagation differential phase shift, neither is $\xi(\gamma)$. The relationship among the parameters in (10c) is illustrated in Fig. 1. Measurements of $\rho_{L}$ [or $\left.\operatorname{Re}\left(\nu_{L}\right)\right]$ and $\zeta$ specify a value of $L / \xi(\gamma)$ (or $L / \gamma$ ). However, these measurements alone can not provide unambiguous information about particle canting unless $L$ is also measured.

When expressed in circular polarization measurables $\gamma$ is simply

$$
\gamma=\frac{1}{4}\left(\frac{1-\operatorname{Re}\left(\nu_{R L}\right)}{1+\operatorname{Re}\left(\nu_{R L}\right)}\right)
$$

This only requires the measurement of $\nu_{R L}$. For completely oriented hydrometeors $\gamma=0$ and $\operatorname{Re}\left(\nu_{R L}\right)=1$, while $\gamma \rightarrow 0.25$ as $\operatorname{Re}\left(\nu_{R L}\right) \rightarrow 0$ as the hydrometeors become more and more disoriented.

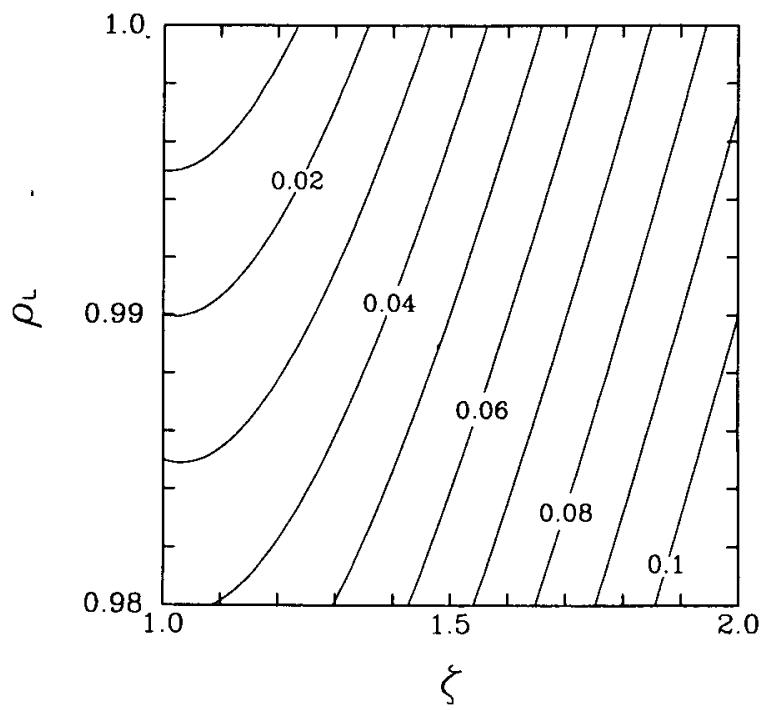

FIG. 1. Contours of the ratio $L / \gamma$ where $L$ is the antilog of $L D R$ and $\gamma$ is the canting parameter defined in the text. $\zeta$ is the antilog of $Z_{D R}$ while $\rho_{L}$ is the magnitude of the cross-correlation $\left(\nu_{L}\right)$ between horizontal and vertical copolarization measurements. 
From these expressions it is clear that, in principle, linear polarization parameters can be calculated from circular polarization measurements. In practice, however, it may often be necessary to correct for propagation differential phase shift first. Recently, McGuinness and Holt (1986) have proposed one method for removing the influence of propagation differential phase shift from estimates of $\zeta$ using circular polarization measurements.

\section{2) Circular polarization Parameters From LINEAR POLARIZATION MEASUREMENTS}

Two particularly useful circular polarization parameters are $\Gamma$, defined by $(9)$, and $\rho_{c}$, defined in (5d).
The circular depolarization ratio can be written in terms of linear polarization parameters as

$$
\Gamma=\frac{\zeta+1+4 \zeta L-2 \zeta^{0.5} \operatorname{Re}\left(\nu_{L}\right)}{\zeta+1+2 \zeta^{0.5} \operatorname{Re}\left(\nu_{L}\right)}
$$

Obviously measurements of $\Gamma$ can be affected by propagation differential phase shift through $\operatorname{Re}\left(\nu_{L}\right)$. On the other hand, by using linear polarization measurements of $\zeta, L$ and $\rho_{L}, \Gamma$ can be well estimated from

$$
\xi(\Gamma)=\frac{\zeta+1+4 \zeta L-2 \zeta^{0.5} \rho_{L}}{\zeta+1+2 \zeta^{0.5} \rho_{L}}
$$

Since all of the terms on the right-hand side are not affected by propagation differential phase shift, neither is $\xi(\Gamma)$. Similarly, the magnitude of $\rho_{c}$ can be written as

$$
\rho_{c}=\frac{\begin{array}{c}
\left(\left\{1-\zeta-2(\zeta L)^{0.5} \operatorname{Im}\left\langle\nu_{V V H V}\right\rangle-2 \zeta L^{0.5} \operatorname{Im}\left(\nu_{H H H V}\right)\right\}^{2}\right. \\
\left.+4\left\{\zeta^{0.5} \operatorname{Im}\left(\nu_{L}\right)+\zeta L^{0.5} \operatorname{Re}\left(\nu_{H H H V}\right)+\zeta^{0.5} \operatorname{Re}\left(\nu_{V V H V}\right)\right\}^{2}\right)^{0.5}
\end{array}}{\left(\left\{1+\zeta+4 L \zeta-2 \zeta^{0.5} \operatorname{Re}\left(\nu_{L}\right)\right\}\left\{1+\zeta+2 \zeta^{0.5} \operatorname{Re}\left(\nu_{L}\right)\right\}\right)^{0.5}},
$$

where $\nu_{H H H V}$ and $\nu_{V V H V}$ are the cross-correlations between the co- and cross-polarized returns which are probably very small even when propagation differential phase shift is present as discussed in subsection $2 a$. Equation (13a) then simplifies to

$$
\rho_{c}=\frac{\left(\{1-\zeta\}^{2}+4 \zeta \operatorname{Im}^{2}\left(\nu_{L}\right)\right)^{0.5}}{\left(\left\{1+\zeta+4 L \zeta-2 \zeta^{0.5} \operatorname{Re}\left(\nu_{L}\right)\right\}\right.} .
$$

For Rayleigh-Gans scatterers, $\operatorname{Im}\left(\nu_{L}\right)$ will usually be small unless there is substantial propagation phase shift (Jameson and Mueller, 1985). However, an estimate of $\rho_{c}$ unaffected by propagation phase changes can be calculated from

$\xi\left(\rho_{c}\right)=\frac{|\zeta-1|}{\left(\left\{\zeta+1+2 \zeta^{0.5} \rho_{L}\right\}\left\{\zeta+1+4 \zeta L-2 \zeta^{0.5} \rho_{L}\right\}\right)^{0.5}}$.

In order to compute $\xi(\Gamma)$ and $\xi\left(\rho_{c}\right),(12)$ and (13) show that it is necessary to measure $\zeta, L$ and $\rho_{L}$. For many radar systems only $\zeta$ and $\rho_{L}$ can be measured. In such cases it is possible to estimate only the product $\Gamma \rho_{c}{ }^{2}$ as illustrated in Fig. 2. Obviously over a wide range of $\zeta$, the estimate of $\Gamma \rho_{c}{ }^{2}$ is not very sensitive to $\rho_{L}$. Hence, if propagation effects were negligible, a measurement of $\Gamma \rho_{c}^{2}$ from a circular polarization radar could be used to estimate $\zeta$ with only a slight uncertainty introduced by the unknown $\rho_{L}$.

Similarly, for radar systems capable of measuring only $L$ and $\zeta$, it is only possible to estimate the quantity $\Gamma \rho_{c}^{2} /(1+\Gamma)^{2}$. The relationship of this product to $L$ and $\zeta$ is illustrated in Fig. 3. Note that this estimate becomes a function only of $\zeta$ when $L$ is sufficiently small. Hence, if $\rho_{c}$ and $\Gamma$ were measured using a circular polarization radar, then $\Gamma \rho_{c}^{2} /(1+\Gamma)^{2}$ provides an estimate of $\zeta$ when $L$ and propagation differential phase shift are sufficiently small.
Equations (8)-(13) demonstrate that parameters of either of the two most commonly used polarizations can be transformed into one another. Provided that propagation effects are small or have been removed, the task, then, is not so much to measure parameters as to find an appropriate theoretical framework for interpreting them meteorologically. For an important but not all inclusive class of scatterers such a framework exists.

For radars operating at long wavelengths, many hydrometeors are Rayleigh-Gans scatterers with shapes adequately represented by symmetric spheroids (see the

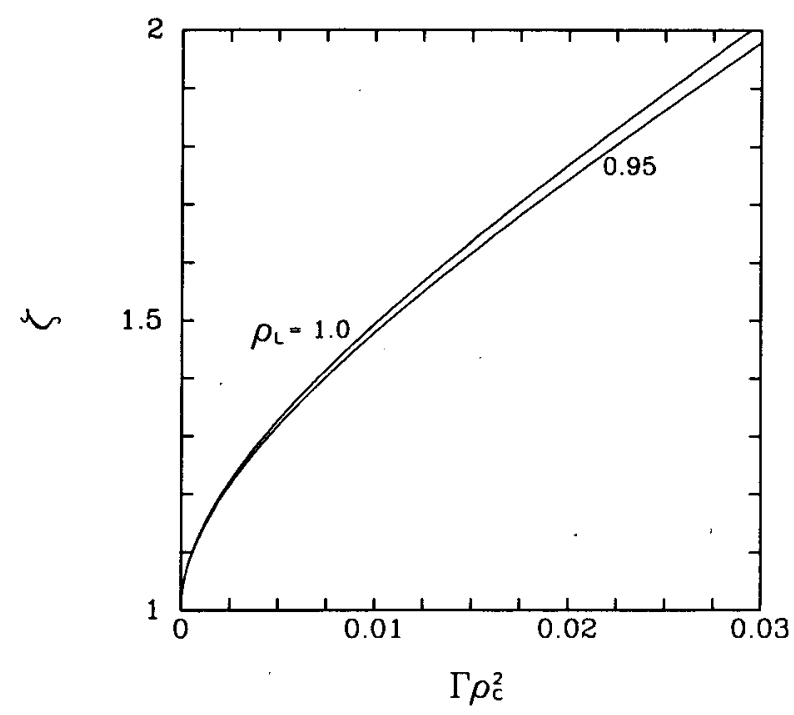

FIG. 2. An illustration for two values of $\rho_{L}$ of the relation between $\zeta$ and the $\Gamma \rho_{c}^{2}$ where $\Gamma$ is the antilog of $C D R$ and $\rho_{c}$ is the magnitude of the cross correlation between the co- and cross-circularly polarized backscattered signals. 


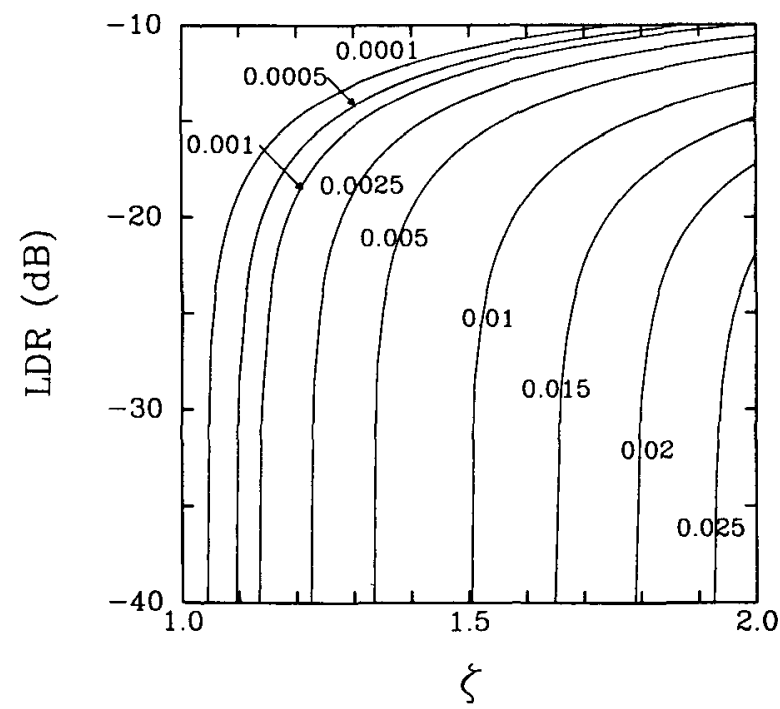

FIG. 3. Contours of $\Gamma \rho_{c}{ }^{2} /(1+\Gamma)^{2}$ as a function of $L D R$ and the antilog of $Z_{D R}(\zeta)$.

discussion in Jameson, 1985, for example). For these hydrometeors polarization measurements can be interpreted in terms of $R, \sigma_{r}$ and $\sigma_{\alpha}$ provided the appropriate algorithms exist for estimating these quantities. In the next section, such algorithms using either linear and circular polarization parameters are presented. In particular the set of linear polarization estimators is completed with the development of an algorithm for estimating $\sigma_{r}$ based on an interpretation of $\rho_{L}$. Potentially this estimator can avoid the problem of propagation differential phase shift which in heavy rain may often plague estimates using circular polarization measurements.

\section{Algorithms for $\mathbf{R}$ and $\sigma_{r}$}

Suppose that there were an ensemble of perfectly oriented raindrops with all the symmetry axes pointed vertically. In addition, suppose that these raindrops are approximately oblate spheroids. For each drop Jameson (1983) showed that for Rayleigh-Gans scattering

$$
\frac{\left|S_{H H}\right|}{\left|S_{V V}\right|}=r^{-7 / 6}
$$

where $r$ is the axis ratio (the smallest axis divided by the largest). Using the definition of $\zeta$, Jameson (1983) then showed that

$$
\zeta^{-1}=\overline{r^{7 / 3}},
$$

where the bar denotes a reflectivity factor weighted average over the entire drop size distribution. Defining

$$
R=\bar{r},
$$

Jameson (1983) also demonstrated that in practice a good estimate of $R$ is given by

$$
\xi(R)=\zeta^{-3 / 7} \text {. }
$$

Using these relations it is possible to interpret $\rho_{L}$ meteorologically. In particular Jameson and Mueller (1985) showed that

$$
\rho_{L}=\frac{\sum_{i}\left|S_{H H}\right|_{i}\left|S_{V V}\right|_{i}}{\left(\sum_{i}\left|S_{H H}\right|_{i}{ }^{2} \sum_{i}\left|S_{V V}\right|_{i}^{2}\right)^{0.5}},
$$

where the summation is over the ensemble of raindrops. Using (14) this expression can be written as

$$
\begin{aligned}
\rho_{L} & =\frac{\overline{r^{7 / 6}}}{\overline{r^{7 / 3} 3^{0.5}}} \\
& =\zeta^{0.5} \overline{r^{7 / 6}},
\end{aligned}
$$

where the last equality is derived from (15a). From this expression another estimator of $R$ can be formed, namely

$$
\xi(R)=\left(\zeta^{-0.5} \rho_{L}\right)^{6 / 7} .
$$

Obviously $\rho_{L}$ is a measure of the ratio of the mean to the root mean square (rms) value of the reflectivity weighted $r^{7 / 6}$. Hence, $\rho_{L}$ is related to the variance of the reflectivity weighted $r^{7 / 6}$. In particular, since

it follows that

$$
\overline{r^{7 / 3}}=\overline{\left(r^{7 / 6}\right)^{2}}
$$

$$
\sigma_{r^{7 / 6}}{ }^{2}=\overline{\left(r^{7 / 6}\right)^{2}}-\overline{r^{7 / 6^{2}}}
$$

where $\sigma^{2}$ denotes the reflectivity weighted variance. Using (15) and (17), (20) can be rewritten as

$$
\sigma_{r}{ }^{2}{ }^{2}=\zeta^{-1}\left(1-\rho_{L}^{2}\right) \text {. }
$$

This dependence of $\rho_{L}$ on the breadth of the distribution of axis ratios can be understood physically. As $\sigma_{r}$ increases it becomes more likely that different parts of the particle distribution will be contributing more backscattered signal at, say, the horizontal polarization than at vertical. This must lead to a reduction in $\rho_{L}$ as can be seen qualitatively by considering an ensemble of scatterers which are initially spherical. Suppose at a later time that some of the scatterers are flattened into very thin oblates which then only contribute backscattered power at horizontal polarization. When all the particles were spheres, $\sigma_{r}=0$ and $\rho_{L}$ was unity since all drops contributed equally to the sum $\Pi$ $=\Sigma\left|S_{H H} \| S_{V V}\right|$ in (16). After modifying the shapes of some of the scatterers, however, $\sigma_{r}>0$ while $\Pi$ is reduced since it is only those particles which contribute to both $E_{H H}$ and $E_{V V}$ which are included in $\Pi$. (This reduction is further enhanced by the increase in the denominator of (16) as a consequence of changes in some of the particle shapes.) Hence, while $\sigma_{r}$ is increased, $\rho_{L}$ is reduced.

Equation (21) shows that $\rho_{L}$ and $\zeta$ provide a measure of the reflectivity weighted variance of $r^{7 / 6}$ which is closely related to $\sigma_{r}^{2}$. While, in general, a direct translation of (21) into an estimate of the reflectivity weighted variance of $r$ is not possible without knowing the axis ratio distribution over the ensemble of drops, 
a reasonable estimate of $\sigma_{r}$ using $\zeta$ and $\rho_{L}$ can still be readily constructed.

In particular, as long as the relative dispersion of the distribution of axis ratios is small,

$$
\overline{r^{2}} \approx{\overline{r^{7 / 3}}}^{6 / 7}
$$

while from the definition of variance:

$$
\sigma_{r}^{2}=\overline{r^{2}}-\bar{r}^{2} \text {. }
$$

Using (18) and (22), (23a) becomes

$$
\xi\left(\sigma_{r}^{2}\right)=\zeta^{-6 / 7}\left(1-\rho_{L}^{12 / 7}\right),
$$

so that $\sigma_{r}$ can be estimated solely from linear polarization measurements.

Interestingly (23b) is analogous to the estimator of $\sigma_{r}$ using circular polarization parameters. It has been shown (Jameson, 1983) that for the ensemble of drops considered in this section

$$
\begin{aligned}
\Gamma & =C\left[(1-R)^{2}+\sigma_{r}{ }^{2}\right] \\
\rho_{c}{ }^{2} & =\frac{(1-R)^{2}}{\left[(1-R)^{2}+\sigma_{r}^{2}\right]}
\end{aligned}
$$

so that

$$
\sigma_{r}^{2}=C^{-1}\left(1-\rho_{c}^{2}\right) \Gamma,
$$

where $C=0.486$ for a wavelength of $10.71 \mathrm{~cm}$. In the next section the estimates of $\sigma_{r}$ using (23b) and (24c) will be compared.

Equations (14)-(24) provide a meteorological format for discussing the results of numerical calculations for canted hydrometeors viewed over a range of radar elevation angles. Before the effects of particle canting and radar elevation can be investigated, however, it is necessary to express the appropriate radar measurables as functions of these two variables. This is done in the next section.

\section{Radar measurables as functions of particle canting angles $(\alpha)$ and radar elevation angle $(\epsilon)$}

In order to compute the variation of both linear and circular polarization parameters as functions of particle canting and radar elevation angle, it will be shown in this section that it is only necessary to write $\boldsymbol{S}_{L}$ in terms of $\alpha$ and $\epsilon$. If $\phi$ is the angle between the direction of propagation of the transmitted wave and the direction $\Xi$ of the symmetry axis of a spheroidal particle and if $\beta$ is the angle between the direction of the vertical polarization vector and the projection of $\Xi$ onto the plane normal to the direction of propagation, then in the Rayleigh-Gans scattering regime (Atlas et al., 1953; Barge, 1972)

$$
S_{H H}=A S_{H H}^{0}+B S_{V V}^{0}
$$

$$
\begin{aligned}
S_{V V} & =C S_{V V}^{0}+D S_{H H}^{0} \\
S_{H V} & =S_{V H} \\
& =\left(S_{V V}^{0}-S_{H H}^{0}\right)(B C)^{0.5},
\end{aligned}
$$

where

$$
\begin{aligned}
& A=\sin ^{2} \phi \cos ^{2} \beta+\cos ^{2} \phi \\
& B=\sin ^{2} \phi \sin ^{2} \beta \\
& C=\sin ^{2} \phi \cos ^{2} \beta \\
& D=\sin ^{2} \beta \sin ^{2} \phi+\cos ^{2} \phi,
\end{aligned}
$$

and the symbol 0 refers to the backscatter matrix elements of the particle when $\epsilon$ and $\alpha$ are both zero.

Angles $\beta$ and $\phi$ can be expressed in terms of $\alpha, \epsilon$, and an angle $\theta$ between the projection of $\Xi$ on the plane perpendicular to local true vertical $\mathbf{V}$ and the horizontal polarization vector which is always assumed to be perpendicular to V. Specifically from Jameson (1985, 1986)

$A=\cos ^{2} \alpha+\sin ^{2} \alpha \sin ^{2} \theta+\sin 2 \epsilon \sin \theta$

$B=\sin ^{2} \alpha \cos ^{2} \theta$

$C=\cos ^{2} \alpha \cos ^{2} \epsilon+\sin ^{2} \alpha \sin ^{2} \epsilon \sin ^{2} \theta+\frac{1}{2} \sin 2 \epsilon \sin 2 \alpha \sin \theta$

$D=\sin ^{2} \alpha\left(\cos ^{2} \theta+\cos ^{2} \epsilon \sin ^{2} \theta\right)+\sin ^{2} \epsilon \cos ^{2} \alpha$

$$
+\frac{1}{2} \sin 2 \epsilon \sin 2 \alpha \sin \theta \text {. }
$$

Combining (5), (25) and (27), it follows for linear polarizations that

$$
\begin{aligned}
\left\langle E_{H H} E_{H H}^{*}\right\rangle=\overline{A^{2}} \sum_{i}\left|S_{H H}^{0}\right|_{i}^{2} & +\overline{B^{2}} \sum_{i}\left|S_{V V}^{0}\right|_{i}^{2} \\
& +2 \overline{A B} \operatorname{Re}\left\{\sum_{i}\left(S_{H H}^{0} S_{V V}^{0 *}\right)_{i}\right\}
\end{aligned}
$$$$
\left\langle E_{V V} E_{V V}^{*}\right\rangle=\overline{D^{2}} \sum_{i}\left|S_{H H}^{0}\right|_{i}^{2}+\overline{C^{2}} \sum_{i}\left|S_{V V}^{0}\right|_{i}^{2}
$$$$
+2 \overline{C D} \operatorname{Re}\left\{\sum_{i}\left(S_{H H}^{0} S_{V V}^{0 *}\right)_{i}\right\}
$$$$
\left\langle E_{H V} E_{H V}^{*}\right\rangle=\overline{B C} \sum_{i}\left|S_{V V}^{0}-S_{H H}^{0}\right|_{i}^{2}
$$

$$
\begin{aligned}
\operatorname{Re}\left\langle E_{H H} E_{V V}^{*}\right\rangle=\overline{A D} \sum_{i}\left|S_{H H}^{0}\right|_{i}^{2}+\overline{B C} \sum_{i}\left|S_{V V}^{0}\right|_{i}^{2} \\
+(\overline{A B}+\overline{B D}) \operatorname{Re}\left\{\sum_{i}\left(S_{H H}^{0} S_{V V}^{0 *}\right)_{i}\right\}
\end{aligned}
$$

$\operatorname{Im}\left\langle E_{H H} E_{V V}^{*}\right\rangle=(\overline{A C}-\overline{B D}) \operatorname{Im}\left\{\sum_{i}\left(S_{H H}^{0} S_{V V}^{0 *}\right)_{i}\right\}$.

Similarly for transmitted right-hand circular polarization in the absence of propagation phase shifts

$$
\begin{aligned}
& \left\langle E_{R L} E_{R L}^{*}\right\rangle \\
& \left.=\frac{1}{4}\left(\overline{A^{2}}+2 \overline{A D}+\overline{D^{2}}\right) \sum_{i}\left|S_{H H}^{0}\right|_{i}^{2}+\frac{1}{4}\left(\overline{B^{2}}+2 \overline{B C}+\overline{C^{2}}\right) \sum_{i}\left|S_{V V}^{0}\right|_{i}^{2}+\frac{1}{2} \overline{(\bar{B}}+\overline{A C}+\overline{B D}+\overline{C D}\right) \operatorname{Re}\left(\sum_{i}\left(S_{H H}^{0} S_{V V}^{0 *}\right)_{i}\right)
\end{aligned}
$$




$$
\begin{gathered}
\left\langle E_{R R} E_{R R}^{*}\right\rangle=\frac{1}{4}\left(\overline{C^{2}}+2 \overline{B C}+\overline{B^{2}}\right) \sum_{i}\left|S_{V V}-S_{H H}\right|_{i}^{2} \\
\left\langle E_{R L} E_{R R}^{*}\right\rangle=\frac{1}{4}\left(\overline{C^{2}}-\overline{B^{2}}\right) \sum_{i}\left|S_{V V}^{0}\right|_{i}^{2}-\frac{1}{4}\left(\overline{A^{2}}-\overline{D^{2}}\right) \sum_{i}\left|S_{H H}^{0}\right|_{i}^{2}+\frac{1}{4}\left(\overline{A^{2}}-\overline{D^{2}}\right) \sum_{i}\left(S_{H H}^{0} S_{V V}^{0 *}\right)_{i}-\frac{1}{4}\left(\overline{C^{2}}-\overline{B^{2}}\right) \sum_{i}\left(S_{H H}^{0} S_{V V}^{0 *}\right)_{i} .
\end{gathered}
$$

Although (29) may be used to calculate circular polarization quantities, (5) suggests that (28) and (29) may be combined so that

$$
\begin{aligned}
\left\langle E_{R L} E_{R L}^{*}\right\rangle=\frac{1}{4}\left\{\left\langle E_{H H} E_{H H}^{*}\right\rangle\right. & +\left\langle E_{V V} E_{V V}^{*}\right\rangle \\
& \left.+2 \operatorname{Re}\left\langle E_{H H} E_{V V}^{*}\right\rangle\right\} \\
\left\langle E_{R R} E_{R R}^{*}\right\rangle= & \frac{1}{4}\left\{\left\langle E_{H H} E_{H H}^{*}\right\rangle+\left\langle E_{V V} E_{V V}^{*}\right\rangle\right. \\
& \left.-2 \operatorname{Re}\left\langle E_{H H} E_{V V}^{*}\right\rangle+4\left\langle E_{H V} E_{H V}^{*}\right\rangle\right\} \\
\left\langle E_{R L} E_{R R}^{*}\right\rangle= & \frac{1}{4}\left\{\left\langle E_{V V} E_{V V}^{*}\right\rangle-\left\langle E_{H H} E_{H H}^{*}\right\rangle\right. \\
& \left.+2 j \operatorname{Im}\left\langle E_{H H} E_{V V}^{*}\right\rangle\right\} .
\end{aligned}
$$

Hence, it is only necessary to evaluate (28) for linear polarization and then to use (30) to derive the circular polarization parameters.

Equations (28) and (30) can now be used to investigate the behavior of the linear and circular polarization parameters as $\sigma_{\alpha}$ and $\epsilon$ change. From these expressions it is apparent, however, that the exact response of polarization parameters to changes in $\sigma_{\alpha}$ or $\epsilon$ will depend upon the distributions of the sizes, the shapes, and the refractive indices of the hydrometeors. Results from a particular example can only be generalized in a qualitative sense.

In the next section polarization parameters are calculated for a distribution of canted hydrometeors. When evaluating the coefficients in (28), it will be assumed that the distributions of $\phi$ and $\alpha$ are identical for all the scatterers. Although in reality this is not likely to be strictly correct, such an assumption is necessary since the distributions of these angles as functions of particle sizes are unknown. This assumption is also sufficient for a numerical demonstration.

\section{An example}

The purpose of this section is not to model a particular type of hydrometeor but to provide a qualitative example of the effects of particle canting and radar elevation angle on polarization measurements. Computations will be made for a distribution of raindrops. Real rain, however, is a highly oriented medium (McCormick et al., 1972) characterized by small $\sigma_{\alpha}$ (Hendry et al., 1987). In order to amplify the effect of radar elevation angle in the computations, an artificially enlarged $\sigma_{\alpha}$ will be used.

Before proceeding with the computations, initial conditions need to be specified. The subsequent calculations will exclude any propagation effects.

\section{a. Assumptions}

\section{1) DROP SIZE DISTRIBUTION}

The drop size distribution selected for this example corresponds to one which might be found in a thunderstorm (Sekhon and Srivastava, 1971) with a rainfall rate of $15 \mathrm{~mm} \mathrm{~h}^{-1}$. The drops are allowed to range in size from $0.01-0.6 \mathrm{~cm}$ equivalent spherical diameter, and they are assumed to be equilibrium shaped (Pruppacher and Pitter, 1971).

\section{2) CANTing ANGLE Distribution}

The spatial distribution of particle canting angles is inherently three dimensional. Hence, the canting angle distribution can be expressed as a function of solid angle at an angle $\alpha$ from the vertical and an azimuth angle around the unit circle on a plane bisecting the unit sphere. This description can be further simplified by assuming that $\alpha$ is distributed axisymmetrically about the vertical. This distribution will be indicated by $P_{\Omega}(\alpha)$ where $P$ is the probability density function and $\Omega$ denotes the solid angle.

Since almost nothing is known about the distribution of particle canting angles, there is great freedom to choose practically any form for $P_{\mathrm{g}}(\alpha)$. Since symmetric hydrometeors tend to orient themselves with respect to the gravitational field so that the axes of symmetry are at or near vertical, a natural choice is a distribution in which $P_{\Omega}(\alpha)$ is a maximum at $\alpha=0$. An axisymmetric Gaussian distribution is a particularly convenient selection. By increasing the variance, the Gaussian distribution smoothly transforms into the uniform distribution for which the scattering is isotropic. This latter distribution is particularly appropriate for tumbling hydrometeors such as large hail.

In addition there is a mathematical advantage to using a Gaussian $P_{\Omega}(\alpha)$. The circular polarization parameters depend upon the angle $\beta$ between the direction of the vertical polarization vector and the projection of the direction of the symmetry axis onto the plane normal to the direction of propagation. It can be shown analytically for a uniform distribution and numerically for a Gaussian distribution that $P_{\Omega}(\alpha)$ yields an equivalent uniform or Gaussian distribution of $P(\beta)$. Thus, for example, if the variance of $P(\beta)$ is estimated from circular polarization observations, then $\sigma_{\alpha}$ of $P_{\Omega}(\alpha)$ is known. 
Other forms of $P_{\Omega}(\alpha)$, however, are possible. A family of $P_{\Omega}(\alpha)$ can be defined through the relation

$$
P_{\Omega}(\alpha)=\frac{2 P(\alpha)}{\sin \alpha},
$$

where $P(\alpha) d \alpha$ is the probability of finding $\alpha$ between $\alpha$ and $\alpha+d \alpha$. In order to investigate the sensitivity of polarization parameters to a non-Gaussian form of $P_{\Omega}(\alpha)$, computations using a normal distribution of $P(\alpha)$ in (31) are also included in the results.

\section{b. Effect of particle canting on polarization parameters}

In Fig. 4 the variation of $\rho_{L}, L D R, Z_{D R}$ and $\Phi_{L}$ are given as functions of increasing variance of the two canting angle distributions $P_{\Omega}(\alpha)$. The radar elevation angle is zero. Both $Z_{D R}$ and the argument of the crosscorrelation function $\left(\Phi_{L}\right)$, which is the average of the difference in the phase shifts due to backscatter between horizontal and vertical polarizations, show the identical systematic decrease with increasing $\sigma_{\alpha}$ for both canting angle distributions. Figure 4 suggests, therefore, that if the propagation differential phase shift is negligible, then $\Phi_{L}$ provides no new information beyond that derived from $Z_{D R}$ for Rayleigh-Gans scatterers.

The difference between the Gaussian $P_{\Omega}(\alpha)$ and that defined by (31) is illustrated in Fig. 4. Both distributions become nearly uniform as $\sigma_{\alpha} \rightarrow 90^{\circ}$. However, a uniform distribution for $P(\alpha)$ corresponds to an infinite $P_{\Omega}(\alpha)$ at $\alpha=0^{\circ}$. Uniform $P(\alpha)$, therefore, does not yield isotropic scattering, and $Z_{D R}$ does not go to $0 \mathrm{~dB}$. On the other hand, when $P_{\mathrm{Q}}(\alpha)$ is uniform, the scattering does become isotropic and $Z_{D R}$ does go to zero.

In contrast to $Z_{D R}, \rho_{L}$ is only slightly reduced by increases in $\sigma_{\alpha}$. The direction of this change can be

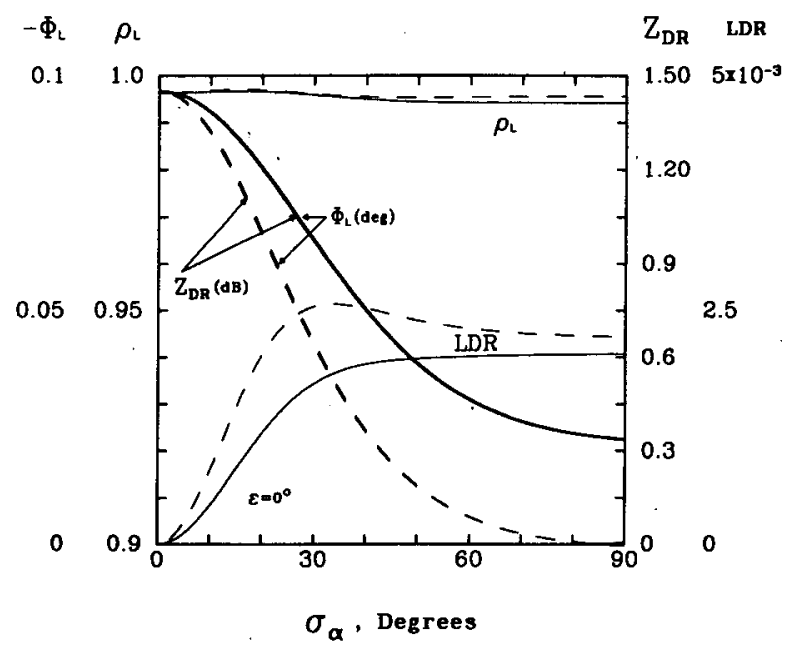

FIG. 4. Linear polarization parameters for a distribution of Rayleigh-Gans scatterers as functions of the standard deviation $\left(\sigma_{\alpha}\right)$ of two distributions of particle canting angles $P_{\Omega}(\alpha)$. The solid lines correspond to a Gaussian $P_{\mathrm{B}}(\alpha)$ while the dashed lines correspond to $P_{\mathrm{s}}(\alpha)$ defined in (31) for a Gaussian $P(\alpha)$. anticipated from qualitative arguments. Imagine an ensemble of dipole scatterers all with $\alpha=45^{\circ}\left(\sigma_{\alpha}\right.$ $=0^{\circ}$ ); then $\rho_{L}$ is unity. Suppose that some of these scatterers are subsequently oriented horizontally. As a consequence $\sigma_{\alpha}>0^{\circ}$ and the sum $\Pi=\sum\left|S_{H H} \| S_{V V}\right|$ in (16) is reduced since there are now fewer scatterers contributing both to horizontally and to vertically polarized backscattered signals. Also while $\sum\left|S_{H H}\right|^{2}$ is enhanced in the process, $\Sigma\left|S_{V V}\right|^{2}$ is decreased so that the denominator in (16) does not substantially change. Hence, $\rho_{L}$ should decrease. For most real hydrometeors, however, the magnitude of this decrease will be small since the hydrometeors contribute nearly equally to $\Pi, \sum\left|S_{H H}\right|^{2}$ and $\sum\left|S_{V V}\right|^{2}$. The signals $E_{H H}$ and $E_{V V}$ generally remain highly correlated even when $\sigma_{\alpha}$ is very large.

The final linear polarization parameter in Fig. 4 is $\operatorname{LDR}(7 \mathrm{~b})$ which is a function of both the hydrometeor shapes and canting. This interdependence can be seen more clearly by rewriting $L D R$ as a function of the canting parameter $\gamma(10)$. From (10c)

$$
L=\frac{\gamma\left(1+\zeta-2 \rho_{L} \zeta^{0.5}\right)}{\zeta}
$$

Although $\gamma$ is only a function of particle canting angles, $\zeta$ is a function of both particle shapes and canting. Therefore, the effects of shape and canting on $L D R$ cannot be separated. An unambiguous interpretation of $L D R$ is, therefore, difficult without some other additional information.

Nevertheless, the approach of $L D R$ toward a constant as $\sigma_{\alpha}$ increases can be understood using (31). As $\sigma_{\alpha}$ increases, $\zeta \rightarrow 1$ so that (32) simplifies to

$$
L \sim 2 \gamma\left[1-\rho_{L}\right]
$$

Since $\gamma$ becomes constant as $\sigma_{\alpha}$ increases [see the discussion of (11)] and since $\rho_{L}$ remains nearly unchanged (Fig. 4), (33) implies that $L D R$ should also increase toward a constant value.

The response of the circular polarization variables to changes in $\sigma_{\alpha}$ is shown in Fig. 5. Although $\Phi_{c}$ is insensitive to $\sigma_{\alpha}$ both $\rho_{c}$ and $\Gamma$ decrease as $\sigma_{\alpha}$ increases. While $\rho_{c} \rightarrow 0$ as $\sigma_{\alpha} \rightarrow 90^{\circ}, \Gamma$ does not. Since $\zeta \rightarrow 1$ as $\sigma_{\alpha}$ increases (12a) becomes

$$
\Gamma=\frac{2+4 L-2 \operatorname{Re}\left(\nu_{L}\right)}{2+2 \operatorname{Re}\left(\nu_{L}\right)} .
$$

Here $\Gamma$ will only be zero if both $L \rightarrow 0$ and $\operatorname{Re}\left(\nu_{L}\right) \rightarrow$ 1 , i.e., only if the scatterers are spheres.

Similarly in this limiting case of large $\sigma_{\alpha},(13 b)$ simplifies to

$$
\rho_{c}=\frac{2 \operatorname{Im}\left(\nu_{L}\right)}{\left(\left\{2+4 L-2 \operatorname{Re}\left(\nu_{L}\right)\right\}\left\{2+2 \operatorname{Re}\left(\nu_{L}\right)\right\}\right)^{0.5}} .
$$

In the absence of propagation effects, however,

$$
\begin{aligned}
\operatorname{Im}\left(\nu_{L}\right) & =\rho_{L} \sin \left(\Phi_{L}\right) \\
& =\rho_{L} \sin \left(\overline{\delta_{H}-\delta_{V}}\right)
\end{aligned}
$$




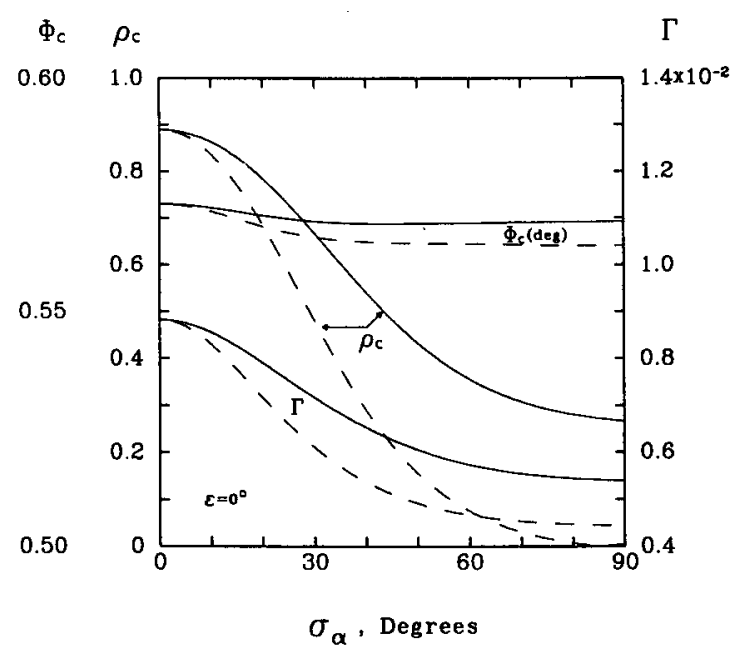

FIG. 5. As in Fig. 4 except circular polarization parameters are shown.

(Jameson and Mueller, 1985) where $\delta_{H}$ and $\delta_{V}$ are the phase shifts induced by backscattering and the overbar denotes an average over all the particles in the sampling volume. Since $P_{\mathrm{\Omega}}(\alpha)$ becomes uniform as $\sigma$ increases, the scattering becomes more and more isotropic so that the sine term in (36) and, therefore, $\rho_{c}$ both go to zero. In this limiting case the oblate scatterers now appear to be spherical. This is a particularly good example illustrating the distinction which should always be made between properties of an ensemble versus those of individual scatterers within the ensemble.

The response of the linear (Fig. 4) and circular (Fig. 5) polarization parameters to changes in $\sigma_{\alpha}$ can also be "explained" in terms of an "apparent" mean axis ratio $R$ and "apparent" standard deviation $\sigma_{r}$ (Fig. 6). The use of apparent is intended to distinguish between the estimates of $R$ and $\sigma_{r}$ deduced by remote sensing which may have been altered by particle canting from the actual or intrinsic values of the particle ensemble. In Fig. $6 R_{\zeta}$ and $R^{\prime}$ are estimates using (15) and (18), respectively. The $\sigma_{c}$ and $\sigma_{L}$ are estimates of $\sigma_{r}$ using circular (24c) and linear (23b) polarization parameters.

As $\sigma_{\alpha}$ increases, the scatterers appear more spherical $\left(R_{\zeta}, R^{\prime} \rightarrow 1\right)$. On the other hand, $\sigma_{c}$ and $\sigma_{L}$ appear initially to increase and then to level off. Thus because of the relatively small increase in the apparent $\sigma_{r}$, the substantial decrease of both $\rho_{c}$ and $\Gamma$ (Fig. 5) must be attributed largely to the rise of the apparent mean axis ratio toward unity. The same remark applies to the response of $\zeta$ (Fig. 4) as well. In contrast both $L$ and $\rho_{L}$ seem most responsive to changes in the apparent $\sigma_{r}$.

\section{c. Effect of radar elevation angle}

The effect of increasing the radar elevation angle on the linear polarization patterns is shown in Fig. 7. The decline of $Z_{D R}$ indicates that the particles appear more

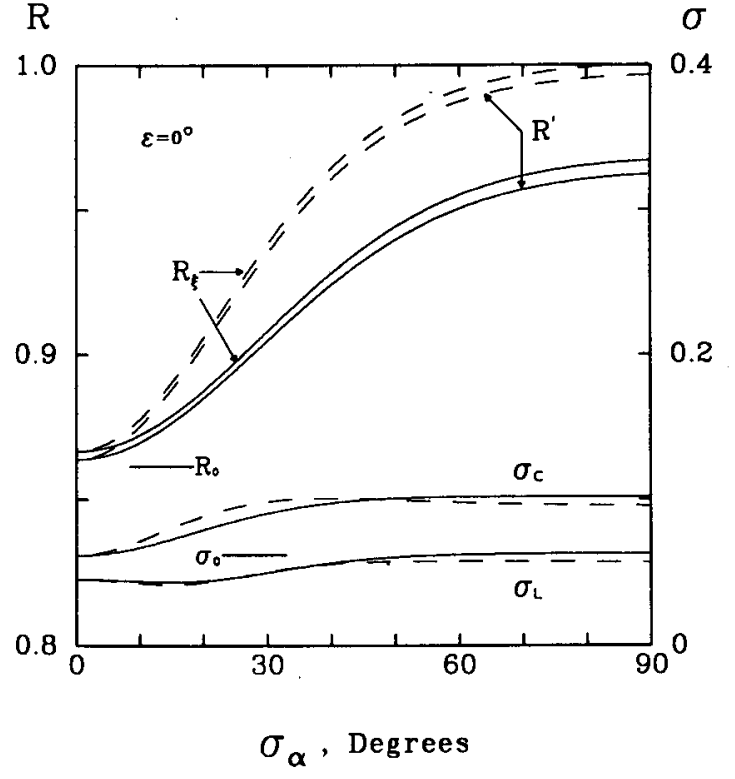

FIG. 6. The reflectivity factor weighted mean axis ratio estimated from $Z_{D R}(R \zeta)$ and a combination of $\rho_{L}$ and $Z_{D R}\left(R^{\prime}\right)$ and the reflectivity weighted standard deviation of $r$ estimated from linear $\left(\sigma_{L}\right)$ and circular $\left(\sigma_{c}\right)$ polarization parameters as functions of $\sigma_{\alpha}$. The solid lines correspond to a Gaussian $P_{\mathrm{n}}(\alpha)$ while the dashed lines correspond to $P_{\mathrm{n}}(\alpha)$ defined in (31) for a Gaussian $P(\alpha) . R_{0}$ and $\sigma_{0}$ are the true values for the model raindrop distribution.

spherical (Fig. 9), while the rise in $\rho_{L}$ can be interpreted as an apparent decrease in $\sigma_{r}$ (see the discussion in section 3 ). $L D R$ becomes very small as $\epsilon$ increases, but it does not disappear even for $\epsilon=90^{\circ}$, since particle canting still produces some linear cross polarization.

From this interpretation of the linear polarization results, one expects that both $\Gamma$ and $\rho_{c}$ should decrease with increasing $\epsilon$ because $\zeta \rightarrow 1$ as $\epsilon \rightarrow 90^{\circ}$, so that

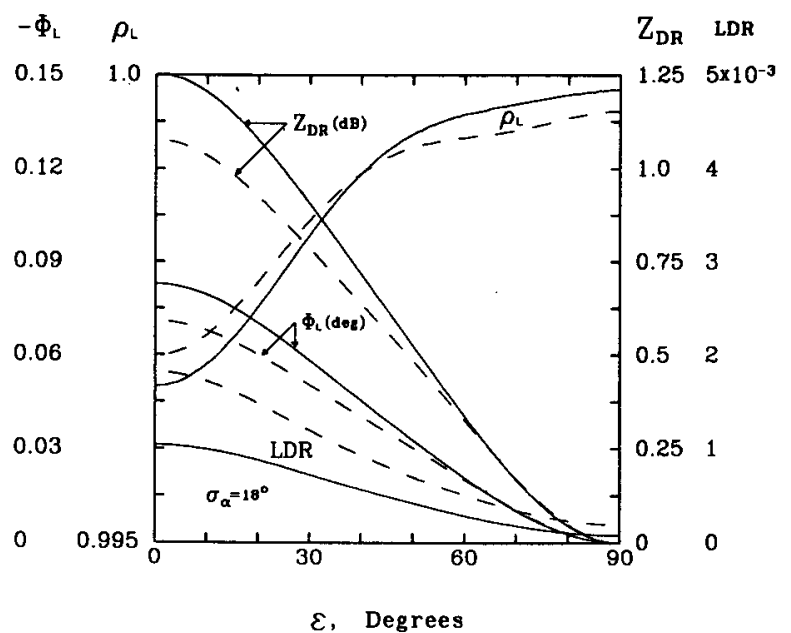

Fig. 7. Linear polarization parameters as functions of the radar elevation angle for the model distribution of scatterers with a Gaussian $P_{\Omega}(\alpha)$ with $\sigma_{\alpha}=18^{\circ}$. 
(34)-(36) apply in this case as well. This is indeed what happens (Fig. 8). The $\Gamma$ does not vanish when $\epsilon=90^{\circ}$ since $L$ remains greater than zero and $\operatorname{Re}\left(\nu_{L}\right) \neq 1$ exactly. On the other hand, at vertical incidence $\rho_{c}$ does vanish since $\Phi_{L} \rightarrow 0$ as $\epsilon \rightarrow 90^{\circ}[(35)-(36)]$. Interestingly, however, $\rho_{c}$ is essentially unaffected until $\epsilon$ reaches about $50^{\circ}$.

In summary it appears that the effects of increasing elevation angle and increasing variance of particle canting are quite similar. This is most obvious in that both cause $\zeta \rightarrow 1$; i.e., both factors tend to make the backscattering increasingly isotropic. However, the effects are not identical. While the apparent variance of the axis ratio increases with increasing $\sigma_{\alpha}$, it decreases with increasing $\epsilon$ (Fig. 9). In other words the horizontally and vertically polarized signals are more highly correlated as $\epsilon$ increases, but less correlated as $\sigma_{\alpha}$ increases. In this sense increasing the radar elevation angle leads to more isotropic scatter than does an increase in particle canting.

\section{Summary}

In this paper some of the mathematical interrelationships between parameters measured using either circular or linear polarizations are investigated. In particular, methods are developed for estimating differential reflectivity $(\zeta)$, linear depolarization ratio $(L)$, the magnitude of the cross correlation between horizontal and vertical copolarizations $\left(\rho_{L}\right)$, and the canting parameter $(\gamma)$ from circular polarization measurements when propagation differential phase shift is negligible or has been removed. Conversely, it is shown that the circular depolarization ratio $(\Gamma)$ and the magnitude of the cross correlation between co- and cross-circularly polarized backscattered signals $\left(\rho_{c}\right)$ can be estimated from linear polarization measurements.

For prolate and oblate spheroids which are in the Rayleigh-Gans scattering regime (radar wavelength

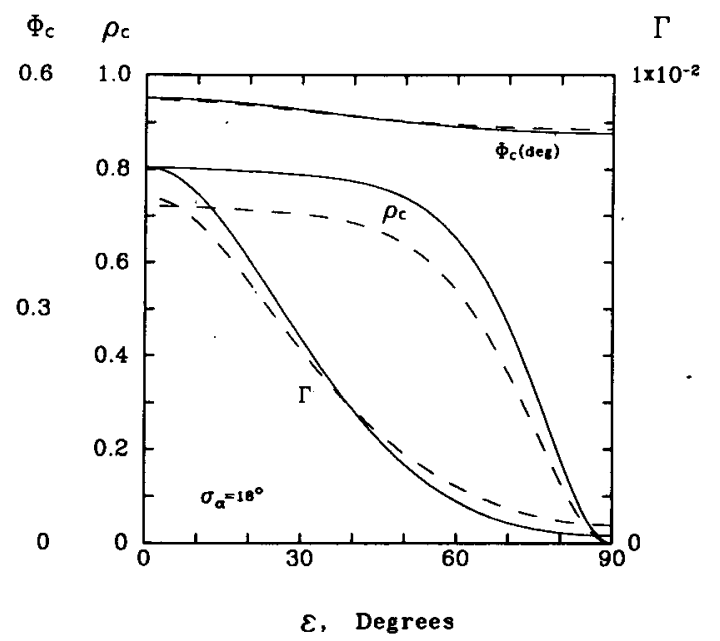

Fig. 8. Circular polarization parameters as functions of the radar elevation angle for the model distribution of scatterers with $\sigma_{\alpha}=18^{\circ}$.
$\mathrm{R}$

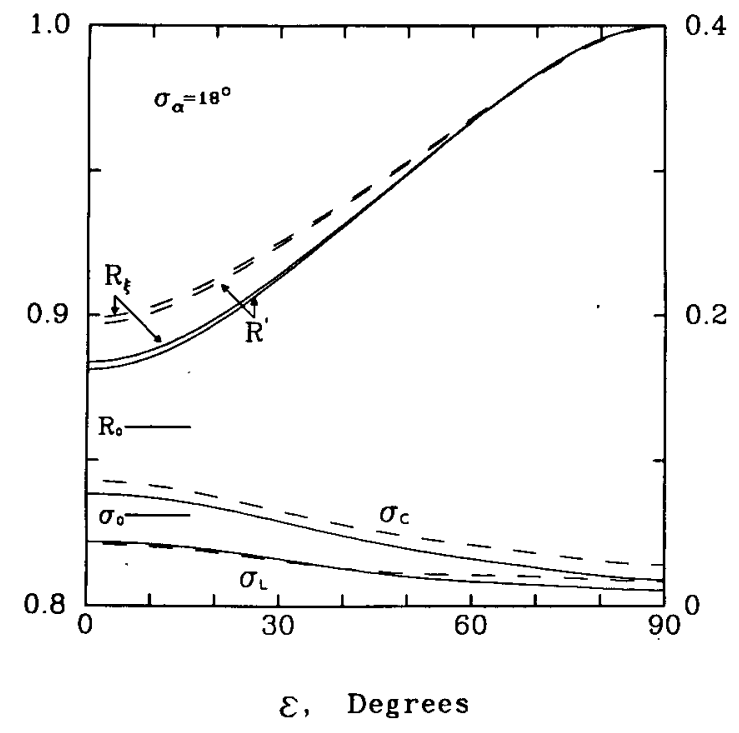

FIG. 9. As in Fig. 6 except that the estimates are functions of the radar elevation angle.

much larger than the index of refraction weighted particle size), these measurements can be interpreted in terms of the reflectivity weighted mean axis ratio $(R)$, standard deviation of the reflectivity weighted axis ratio distribution $\left(\sigma_{r}\right)$ of the ensemble of scatterers, and the standard deviation $\left(\sigma_{\alpha}\right)$ of the distribution of canting angles (the angle between local vertical and the particle symmetry axis). In particular it is shown that $\rho_{L}$, which remains large even when particle canting is substantial, is a function of both $R$ and $\sigma_{r}$ which, when combined with $\zeta$, can be used to estimate $\sigma_{r}$. However, when $\sigma_{\alpha}$ can not be estimated, $R$ and $\sigma_{r}$ deduced from polarization measurements must be regarded as "apparent" properties since the actual or "intrinsic" values of the ensemble may have been modified by particle canting. For truly useful measurements it is essential, therefore, to monitor particle canting.

This approach permits a simple qualitative meteorological interpretation of the way both radar elevation angle $(\epsilon)$ and $\sigma_{\alpha}$ affect linear and circular polarization measurements. Specifically, it is demonstrated that for an ensemble of oblate raindrops, increases in both $\epsilon$ and $\sigma_{\alpha}$ cause the apparent reflectivity weighted mean axis ratio to approach unity. On the other hand, an increase in $\sigma_{\alpha}$ increases the apparent $\sigma_{r}$ while the opposite holds for $\epsilon$. Depending upon the relative importance of $R$ and $\sigma_{r}$, it is possible to visualize the effects that changes of $\epsilon$ and $\sigma_{\alpha}$ will have on the various polarization parameters. A more general but less meteorological alternative way of reexpressing these results is that increases in $\epsilon$ and $\sigma_{\alpha}$ both increase the apparent isotropy of the backscattering medium, but that when $\sigma_{\alpha}$ increases, the correlation between measurements at alternating horizontal and vertical copolarizations decreases while the opposite is true for $\epsilon$. Hence, for this 
model distribution of drops, an increase in $\epsilon$ tends to produce greater isotropy than an increase in $\sigma_{\alpha}$.

Acknowledgment. This work was supported by the National Science Foundation under Grant ATM8596007. The author is indebted to Dr. James Metcalf of the Air Force Geophysics Laboratory for his enlightening and detailed review of this manuscript.

\section{REFERENCES}

Atlas, D., M. Kerker and W. Hitschfeld, 1953: Scattering and attenuation by nonspherical atmospheric particles. J. Atmos. Terr. Phys., 3, 108-1 19.

Barge, B. L., 1972: Hail detection with a polarization diversity radar. Stormy Weather Group Sci. Rep. MW-71, Ph.D thesis, McGill University, $80 \mathrm{pp}$.

Hendry, A., Y. M. M. Antar and G. C. McCormick, 1987: On the relationship between the degree of preferred orientation in precipitation and dual-polarization radar echo characteristics. Radio Sci., 22, 37-50.

Jameson, A. R, 1983: Microphysical interpretation of multi-parameter radar measurements in rain. Part I: Interpretation of polarization measurements and estimation of raindrop shapes. $J$. Atmos. Sci., 40, 1792-1802.

- , 1985: On deducing the microphysical character of precipitation from multiple-parameter radar polarization measurements. $J$. Climate Appl. Meteor., 24, 1037-1047.

- 1986: Corrigendum to "On deducing the microphysical char- acter of precipitation from multiple-parameter radar polarization measurements." J. Climate Appl. Meteor., 25, 1075.

$\longrightarrow$, and E. A. Mueller, 1985: Estimation of differential phase shift from sequential orthogonal linear polarization radar measurements. J. Atmos. Oceanic Technol., 2, 133-137.

Maffett, A. L., 1968: Scattering matrices. Methods of Radar CrossSection Analysis, J. W. Crispin and K. M. Siegel, Eds., Academic Press, 425 pp.

McCormick, G. C., 1979: Relationship of differential reflectivity to correlation in dual-polarization radar. Electron. Lett., 15, 265266.

- A. Hendry and B. L. Barge, 1972: The anisotropy of precipitation media. Nature, 238, 214-216.

McGuiness, R., and A. R. Holt, 1986: Interpreting $C D R, Z_{D R}$ radar data using a computer colour graphics display. Proc. 23rd Conf. Radar Meteorology, Boston, Amer. Meteor. Soc., JP337-JP380.

Pruppacher, H. R., and R. L. Pitter, 1971: A semi-empirical determination of the shape of cloud and raindrops. J. Atmos. Sci., 28, 86-94.

Sachidananda, M., and D. S. Zrnić, 1986: Differential propagation phase shift and rainfall rate estimation. Radio Sci., 21, 235247.

Saxon, D. S., 1955: Tensor scattering matrix for the electromagnetic field. Phys. Rev., 100, 1771-1775.

Sekhon, R. S., and R. C. Srivastava, 1971: Doppler radar observations of drop-size distributions in a thunderstorm. J. Atmos. Sci., 28, 983-994.

Sinclair, G., 1948: Modification of the radar equation for arbitrary targets and arbitrary polarizations. Rep. No. 302-19, Antenna Laboratory, Ohio State University, 38 pp. 